Pacific Journal of Mathematics

A MOMENT PROBLEM FOR POSITIVE MEASURES ON THE 


\title{
A MOMENT PROBLEM FOR POSITIVE MEASURES ON THE UNIT DISC
}

\author{
AHARON ATZMON
}

A necessary and sufficient condition on an infinite matrix $\left(\alpha_{m, n}\right)_{m, n=0}^{\infty}$ is given for the existence of a positive Borel measure $\mu$ on the closed unit disc $D$ in the complex plane such that

$$
\alpha_{m, n}=\int_{D} z^{m} \bar{z}^{n} d \mu(z)
$$

holds for all nonnegative integers $m$; $n$.

1. Introduction. The purpose of this note is to present the solution of the following complex moment problem:

For which infinite matrices $\left(\alpha_{m, n}\right)_{m, n=0}^{\infty}$ does there exist a positive Borel measure $\mu$ on the closed unit disc $D$ is the complex plane such that

$$
\alpha_{m, n}=\int_{\boldsymbol{D}} z^{m} \bar{z}^{n} d \mu(z)
$$

holds for all nonnegative integers $m, n$ ?

In $\S 2$ we give the complete solution of this problem, and in $\S 3$ we deduce, as corollaries, known solution of the trigonometric moment problem and the power moment problems for the intervals $[0,1]$ and $[-1,1]$.

Our interest in the moment problem (1.1) arose from the observation that the invariant subspace problem for bounded linear operators on a separable Hilbert space is equivalent to the following problem:

Let $A=\left(\alpha_{m, n}\right)_{m, n=0}^{\infty}$ be an infinite matrix which satisfies the following two conditions:

$$
\sum_{m, n=0}^{\infty} \alpha_{m, n} w_{m} \bar{w}_{n}>0
$$

and

$$
\sum_{m, n=0}^{\infty}\left(\alpha_{m, n}-\alpha_{m+1, n+1}\right) w_{m} \bar{w}_{n} \geqq 0
$$

for every nonzero sequence $\left(w_{n}\right)_{n=0}^{\infty}$ of complex numbers which has only finitely many nonzero terms.

Let $\boldsymbol{P}$ denote the vector space of all polynomials with complex 
coefficients, and define on it the inner product

$$
\langle p, q\rangle=\sum_{m=0}^{M} \sum_{n=0}^{N} \alpha_{m, n} b_{m} \bar{c}_{n}
$$

where $p(z)=\sum_{m=0}^{M} b_{m} z^{m}$ and $q(z)=\sum_{n=0}^{N} c_{n} z^{n}$.

It follows from (1.2) that with this inner product $\boldsymbol{P}$ forms a pre-Hilbert space, and we denote by $\mathscr{H}_{A}$ the Hilbert space obtained as its completion. Let $S$ denote the operator of multiplication by $z$ on $\boldsymbol{P}$, that is, $\operatorname{Sp}(z)=z p(z)$ for $p \in \boldsymbol{P}$. Condition (1.3) implies that $\|S\| \leqq 1$, and therefore, since $P$ is dense in $\mathscr{H}_{A}, S$ admits a unique extension to a bounded linear operator on $\mathscr{H}_{A}$ with the same norm, which we shall also denote by $S$.

The problem which is equivalent to the general invariant subspace problem is as follows:

For every matrix $A=\left(\alpha_{m, n}\right)_{m, n=0}^{\infty}$ which satisfies (1.2) and (1.3) does the associated operator $S$ have a nontrivial invariant subspace?

Indeed, assume that there exists a linear operator $T$ on a separable Hilbert space $\mathscr{H}$, such that $\|T\| \leqq 1$, which has no nontrivial invariant subspace, and let $x$ be any nonzero element of $\mathscr{H}$. Define the matrix $A=\left(\alpha_{m, n}\right)_{m, n=0}^{\infty}$ by: $\alpha_{m, n}=\left\langle T^{m} x, T^{n} x\right\rangle, m, n=0,1,2, \cdots$. The assumptions on $T$ imply that the matrix $A$ satisfies conditions (1.2) and (1.3). Let $S$ be the operator associated with $A$ as before, and consider the isometric linear transformation $U$ of $\mathscr{H}_{A}$ onto $\mathscr{H}$ which is defined on the dense subspace $\boldsymbol{P}$ by $U p=p(T) x, p \in \boldsymbol{P}$. Then $S=U^{-1} T U$, and therefore if $T$ has a nontrivial invariant subspace, neither has $S$.

In view of this observation, it is of interest to consider matrices $\left(\alpha_{m, n}\right)_{m, n=0}^{\infty}$ for which (1.2) and (1.3) hold, and to find further conditions on these matrices which imply that the associated operator $S$ has a nontrivial invariant subspace. In the particular case in which $\left(\alpha_{m, n}\right)_{m, n=0}^{\infty}$ satisfies condition (1.1) for some positive Borel measure $\mu$ on the closed unit disc, then conditions (1.2) and (1.3) are satisfied, and $\mathscr{H}_{A}$ can be identified with $H^{2}(d \mu)$-the closure of $\boldsymbol{P}$ in $L^{2}(d \mu)$ and the associated operator $S$ is now multiplication by $z$ on $H^{2}(d \mu)$. It is worth nothing that the problem of whethere for every positive Borel measure $\mu$ on $\boldsymbol{D}$ the operator of multiplication by $z$ on $H^{2}(d \mu)$ has a nontrivial invariant subspace is still open, and is equivalent to the invariant subspace problem for sub-normal operators. (We learned this from A. Shields. See also [5] p. 192 for further details.)

In the particular cases when $\left(\alpha_{m, n}\right)_{m, n=0}^{\infty}$ is a Toeplitz matrix or a Hankel matrix, which satisfies conditions (1.2) and (1.3), the operator $S$, associated with this matrix, has a nontrivial invariant subspace 
whether or not the moment problem (1.1) is soluble for the matrix $\left(\alpha_{m, n}\right)_{m, n=0}^{\infty}$. This can be seen as follows: Let $l_{0}$ be the polynomial which is identically equal to 1 , and consider the closed subspace $M$ of $\mathscr{H}_{A}$ spaned by the vectors $S^{n} l_{0}, n=0,1,2, \ldots$. If $M \neq \mathscr{H}_{A}$ then $M$ is a nontrivial invariant subspace for $S$, and if $M=\mathscr{H}_{A}$ it is easily verified that in the case of Toeplitz matrices $S$ is unitary and in the case of Hankel matrices $S$ is self-adjoint. Thus by well known results $S$ has a nontrivial invariant subspace also in this case. Finally, we remark that $S$ has obviously a nontrivial invariant subspace when $\left(\alpha_{m, n}\right)_{m, n=0}^{\infty}$ is a diagonal matrix.

2. Solution of the moment problem. The general solution of the moment problem (1.1) is given by the following proposition:

THEOREM 2.1. Let $\left(\alpha_{m, n}\right)_{m, n=0}^{\infty}$ be an infinite matrix of complex numbers. Then condition (1.1) holds for some positive Borel measure $\mu$ on the closed unit disc if and only if the following two conditions are satisfied:

$$
\sum_{m, n, j, k=1}^{\infty} \alpha_{m+j, n+k} c_{n, j} \bar{c}_{m, k} \geqq 0
$$

for every matrix $\left(c_{j, k}\right)_{j, k=0}^{\infty}$ with only finitely many nonzero entries

$$
\sum_{m, n=0}^{\infty}\left(\alpha_{m, n}-\alpha_{m+1, n+1}\right) w_{m} \bar{w}_{n} \geqq 0
$$

for every sequence $\left(w_{n}\right)_{n=0}^{\infty}$ with only finitely many nonzero terms.

Proof. Assume first that $\left(\alpha_{m, n}\right)_{m, n=0}^{\infty}$ satisfies condition (1.1) for some positive Borel measure $\mu$ on the closed unit disc $\boldsymbol{D}$ and let $\left(c_{j, k}\right)_{j, k=0}^{\infty}$ be an infinite matrix such that for some positive integers $M$ and $N, c_{j, k}=0$ if $j>M$ or $k>N$. Consider the polynomials: $P_{n}(z)=\sum_{k=0}^{N} c_{n, k} z^{k}, n=0,1,2, \cdots, M$. It follows that

$$
\sum_{m, n, j, k=0}^{\infty} \alpha_{m+j, n+k} c_{n, j} \bar{c}_{m, k}=\int_{D}\left|\sum_{n=0}^{M} z^{n} \overline{p_{n}(z)}\right| d \mu(z) \geqq 0
$$

which proves that (2.1) holds.

To show that (1.1) implies (2.2) consider a sequence of complex numbers $\left(w_{n}\right)_{n=0}^{\infty}$ which has only finitely many nonzero terms. Then

$$
\begin{aligned}
\sum_{m, n=0}^{\infty} \alpha_{m+1, n+1} w_{m} \bar{w}_{n} & =\int_{\boldsymbol{D}}\left|\sum_{n=0}^{\infty} w_{n} z^{n+1}\right| d \mu(z) \\
& \leqq \int_{D}\left|\sum_{n=0}^{\infty} w_{n} z^{n}\right|^{2} d \mu(z)=\sum_{m, n=0}^{\infty} \alpha_{m, n} w_{m} \bar{w}_{n} .
\end{aligned}
$$

Hence (2.2) holds. 
We turn now to the proof that conditions (2.1) and (2.2) are sufficient for (1.1) to be satisfied for some positive Borel measure $\mu$ on $\boldsymbol{D}$. Observe first that (2.1) implies that for any sequence of complex numbers $\left(w_{n}\right)_{n=0}^{\infty}$ which has only finitely many nonzero terms,

$$
\sum_{m, n=0}^{\infty} \alpha_{m, n} w_{m} \bar{w}_{n} \geqq 0
$$

Indeed, (2.3) is obtained by applying (2.1) with the matrix $\left(c_{j, k}\right)_{j, k=0}^{\infty}$ defined by $c_{n, 0}=w_{n}, n=0,1,2, \cdots$, and $c_{n, k}=0$ for $k=1,2,3, \cdots$, $n=0,1,2, \cdots$

We shall now show that (1.1) holds under the additional assumption that whenever $\left(w_{n}\right)_{n=0}^{\infty}$ is not the zero sequence, the inequality in (2.3) is strict, that is, we assume that $\left(\alpha_{m, n}\right)_{m, n=0}^{\infty}$ satisfies also condition (1.2). It will be clear from the proof that the general case can be established in the same way, using only (2.3), by an obvious quotient space argument.

Let now $\mathscr{H}_{A}$ be the Hilbert space associated with the matrix $\left(\alpha_{m, n}\right)_{m, n=0}^{\infty}$ and let $S$ be the corresponding operator as defined in $\S 1$. It is easily verified that condition (2.1) is equivalent to the condition

$$
\sum_{m, n=0}^{l}\left\langle S^{n} p_{m}, S^{m} p_{n}\right\rangle \geqq 0
$$

for any finite set of polynomials $p_{0}, p_{1}, \cdots, p_{l}$. Since the polynomials are dense in $\mathscr{H}_{A}$ it follows from (2.4) that

$$
\sum_{m, n=0}^{l}\left\langle S^{n} f_{m}, S^{m} f_{n}\right\rangle \geqq 0
$$

for any finite set $f_{0}, f_{1}, \cdots, f_{l}$ in $\mathscr{H}_{A}$. By a theorem of J. Bram [3, Theorem 1], condition (2.5) implies that $S$ is a subnormal operator, that is, $S$ can be extended to a normal operator $K$ on a Hilbert space $\mathscr{H}$ which contains $\mathscr{H}_{A}$ as a closed subspace. Moreover, we may also assume that $\|S\|=\|K\|[3$, p. 81, Lemma 2], and since $\|S\| \leqq 1$ we also have $\|K\| \leqq 1$. Let $E$ be the spectral measure associated with $K$ by the spectral theorem for normal operators [4, p. 71]. Let $l_{0}$ be the element of $\boldsymbol{P}$ which is identically equal to 1 , and consider the positive Borel measure $\mu$ defined by $\mu(B)=\left\langle E(B) l_{0}, l_{0}\right\rangle$ for every Borel set $B$ in the plane. We claim now that this measure satisfies the required conditions.

First, since $\mu$ is supported by the spectrum of $K$ and $\|K\| \leqq 1$, the support of $\mu$ is contained in the closed unit disc. Secondly, it follows from the well-known properties of spectral measures [4, p. 61] that for all $p, q \in \boldsymbol{P}$,

$$
\left\langle p(K) l_{0}, q(K) l_{0}\right\rangle=\int_{D} p(z) \overline{q(z)} d \mu(z),
$$


and therefore since $K$ is an extension of $S$ we obtain that

$$
\alpha_{m, n}=\left\langle S^{m} l_{0}, S^{n} l_{0}\right\rangle=\int_{D} z^{m} z^{\bar{n}} d \mu(z) .
$$

This completes the proof of the theorem.

REMARKs. 1. Since every continuous function in the closed unit disc is a uniform limit of polynomials in $z$ and $\bar{z}$, it follows that the solution of the moment problem (1.1), whenever it exists, is unique.

2. It follows from Theorem 2.1 and the fact that a polynomial in one variable has only finitely many zeros, that the measure $\mu$ which satisfies (1.1) has an infinite support if and only if the inequality (2.1) is strict for every nonzero matrix $\left(c_{j, k}\right)_{j, k=0}^{\infty}$ with only finitely many nonzero entries.

3. One might be tempted to conjecture that condition (2.1) can be replaced by condition (2.3) in the hypothesis of Theorem 2.1. That this is not the case can be seen from the following example: Consider the matrix $\left(\alpha_{n, n}\right)_{m, n=0}^{\infty}$ defined by $\alpha_{0,0}=\alpha_{1,1}=2, \alpha_{n, n}=1$ for $n>1$ and $\alpha_{m, n}=0$ for $m \neq n$. Then conditions (2.2) and (2.3) are satisfied but condition (2.1) is violated by the matrix $\left(c_{j, k}\right)_{j, k=0}^{\infty}$ given by: $c_{0,0}=1$, $c_{1,1}=-2$ and $c_{j, k}=0$ otherwise.

3. Application to the trigonometric and power moment problems. In order to apply Theorem 2.1 to the trigonometric and power moment problems we first need two lemmas.

LEMMA 3.1. Let $\left(\alpha_{m, n}\right)_{m, n=0}^{\infty}$ be a matrix for which there exists a positive measure $\mu$ on the closed unit disc such that (1.1) holds. Then:

(a) If $\left(\alpha_{m, n}\right)_{m, n=0}^{\infty}$ is a Toeplitz matrix, that is, $\alpha_{m, n}=\alpha_{m-n, 0}$ for $m \geqq n$, the support of $\mu$ is contained in the unit circle $\{z:|z|=1\}$.

(b) If $\left(\alpha_{m, n}\right)_{m, n=0}^{\infty}$ is a Hankel matrix, that is, $\alpha_{m, n}=\alpha_{m+n, 0}$, $m, n=0,1,2, \cdots$, then the support of $\mu$ is contained in the interval $[-1,1]$.

(c) If $\left(\alpha_{m, n}\right)_{m, n=0}^{\infty}$ is a diagonal matrix that is $\alpha_{m, n}=0$ for $m \neq n$, then using the representation of $\boldsymbol{D}$ in polar coordinates $\boldsymbol{D}=\{(r, \theta)$, $0 \leqq r \leqq 1,0 \leqq \theta \leqq 2 \pi\}$, we have $\mu=\nu \times d \theta$ where $\nu$ is a positive Borel measure on $[0,1]$, and $d \theta$ denotes Lebesgue masure on $[0,2 \pi)$.

Proof. (a) Using the assumption for $m=n=1$ we see that $\int_{D}\left(1-|z|^{2}\right) d \mu(z)=0$, and therefore since $\mu$ is a positive measure its support is contained in $\{z,|z|=1\}$.

(b) Using again the hypothesis for $m=n=1$ we obtain that 


$$
\int_{D}\left(|z|^{2}-z^{2}\right) d \mu(z)=0
$$

hence writing $z=x+i y$ we infer that $\int_{D} y^{2} d \mu(x, y)=0$. Consequently, since $\mu$ is a positive measure its support is contained in $[-1,1]$.

(c) Let $\nu$ be the positive Borel measure on $[0,1]$ defined by $\nu(B)=2 \pi \mu(B \times[0,2 \pi))$ for every Borel set $B$ in $[0,1]$. We claim that $\mu=\nu \times d \theta$. Since the polynomials in $z$ and $\bar{z}$ are dense in the space of continuous functions on $D$, it suffices to prove that for every pair of nonnegative integers $m, n$ we have:

$$
\int_{D} z^{m} \bar{z}^{n} d \mu(z)=\int_{D} z^{m} \bar{z}^{n} d \nu \times d \theta .
$$

Now for $m=n$, the definition of $\nu$ implies that

$$
\int_{D} z^{m} \bar{z}^{n} d \mu(z)=\int_{D} r^{2 m} d \mu(r, \theta)=\frac{1}{2 \pi} \int_{0}^{1} r^{2 m} d \nu(z)=\int_{D} z^{m} \bar{z}^{n} d \nu \times d \theta
$$

and for $m \neq n$ it follows from the hypothesis and the fact that $\int_{0}^{2 \pi} e^{i k \theta} d \theta=0$ for $k \neq 0$, that both sides of (3.1) vanish. This completes the proof of the lemma.

LEMMA 3.2. (a) Let $\left(b_{n}\right)_{n=\infty}^{\infty}$ be a two sided infinite sequence of complex numbers, and define the Toeplitz matrix $\left(\alpha_{m, n}\right)_{m, n=0}^{\infty}$ by $\alpha_{m, n}=b_{n-m}, m, n=0,1,2, \cdots$ Then $\left(\alpha_{m, n}\right)_{m, n=0}^{\infty}$ satisfies conditions (2.1) and (2.2) if and only if

$$
\sum_{p, q=-\infty}^{\infty} b_{p-q} w_{p} \bar{w}_{q} \geqq 0
$$

holds for any sequence $\left(w_{n}\right)_{n=-\infty}^{\infty}$ with only finitely many nonzero terms.

(b) Let $\left(b_{n}\right)_{n=0}^{\infty}$ be an infinite sequence of complex numbers and define the Hankel matrix $\left(\alpha_{m, n}\right)_{m, n=0}^{\infty}$ by $\alpha_{m, n}=b_{m+n}, m, n=0,1,2, \cdots$. Then $\left(\alpha_{m, n}\right)_{m, n=0}^{\infty}$ satisfies (2.1) and (2.2) if and only if the following two conditions are satisfied:

$$
\sum_{q, p=0}^{\infty} b_{p_{+}} w_{p} \bar{w}_{q} \geqq 0
$$

and

$$
\sum_{p, q=0}^{\infty}\left(b_{p+q}-b_{p+q+2}\right) w_{p} \bar{w}_{q} \geqq 0
$$

for any sequence $\left(w_{n}\right)_{n=0}^{\infty}$ of complex numbers with only finitely many nonzero terms.

(c) Let $\left(b_{n}\right)_{n=0}^{\infty}$ be an infinite sequence of complex numbers and 
define the diagonal matrix $\left(\alpha_{m, n}\right)_{m, n=0}^{\infty}$ by $\alpha_{m, n}=b_{n}$ for $m=n$ and $\alpha_{m, n}=0$ for $m \neq n$. Then $\left(\alpha_{m, n}\right)_{m, n=0}^{\infty}$ satisfies (2.1) and (2.2) if and only if the following two conditions are satisfied:

$$
\sum_{p, q=0}^{\infty}\left(b_{p+q}-b_{p_{+q+1}}\right) w_{p} \bar{w}_{q} \geqq 0
$$

and

$$
\sum_{d, q=0}^{\infty} b_{p+q+1} w_{p} \bar{w}_{q} \geqq 0
$$

for any sequence $\left(w_{n}\right)_{n=0}^{\infty}$ of complex numbers with only finitely many nonzero terms.

Proof. (a ) We show first that (2.1) implies (3.2). Let $\left(w_{n}\right)_{n=-\infty}^{\infty}$ be a sequence with only finitely nonzero terms and consider the matrix $\left(c_{j, k}\right)_{j, k=0}^{\infty}$ defined by: $c_{0, p}=w_{-p}, c_{p, 0}=w_{p}, p=0,1,2, \cdots$, and $c_{j, k}=0$ otherwise. A direct computation then shows that

$$
\sum_{p, q=-\infty}^{\infty} b_{p-q} w_{p} \bar{w}_{\theta}=\sum_{u, n, j, k=0}^{\infty} \alpha_{m+j, n+k} c_{n, j} \bar{c}_{m, k} .
$$

Therefore (3.2) follows from (2.2). Conversely, to show that (3.2) implies (2.2), consider any matrix $\left(c_{i, k}\right)_{j, k=0}^{\infty}$ with only finitely many nonzero entries and put $c_{-n, m}=0, m, n=0,1,2, \cdots$ Defining $w_{p}=\sum_{j=0}^{\infty} c_{p+j, j} p=0, \pm 1, \pm 2, \cdots$, we obtain that

$$
\sum_{m, n, j, k=0}^{\infty} \alpha_{m+j, n+k} c_{n, j} c_{m, k}=\sum_{p, q=-\infty}^{\infty} b_{p-q} w_{p} \bar{w}_{q} .
$$

Hence (3.2) implies (2.1). Finally, since (2.2) is clearly satisfied for any Toeplitz matrix, the proof of (a) is complete.

(b) First notice that (3.4) is equivalent to (2.2) and that (3.3) follows from (2.3) hence from (2.1). It therefore remains to prove that (3.3) implies (2.1). Let $\left(c_{j, k}\right)_{i, k=0}^{\infty}$ be any infinite matrix with only finitely many nonzero entries, and define $w_{p}=\sum_{j=0}^{p} c_{p-j, j}, p=0,1,2$, .... A direct computation then shows that

$$
\sum_{p, q=0}^{\infty} b_{p+q} w_{p} \bar{w}_{q}=\sum_{m, n, j, k} \alpha_{m+j, n+k} c_{n, j} \bar{c}_{m, k} .
$$

Hence (2.1) follows from (3.3).

(c) By adding (3.5) and (3.6) we see that

$$
\sum_{p, q=0}^{\infty} b_{p+q} w_{p} \bar{w}_{q} \geqq 0
$$

for any sequence $\left(w_{p}\right)_{p=0}^{\infty}$ with only finitely many nonzero terms. We claim now that for any such sequence $\left(w_{p}\right)_{p=0}^{\infty}$ we also have 


$$
\sum_{p, q=0}^{\infty} b_{m+p+q} w_{p} \bar{w}_{q} \geqq 0
$$

for every nonnegative integer $m$.

Indeed, assuming first that $m=2 k$ for some integer $k$, it follows that

$$
\sum_{p, q=0}^{\infty} b_{m+p+q} w_{p} \bar{w}_{q}=\sum_{p, q=0}^{\infty} b_{p+q} w_{p-k} \bar{w}_{q-k}
$$

where we define $w_{-n}=0, n=0,1,2, \ldots$. Consequently (3.8) follows from (3.7) for $m$ even. Similarly (3.5) implies (3.8) for $m$ odd.

To prove that (3.8) implies (2.1), consider any matrix $\left(c_{j, k}\right)_{j, k=0}^{\infty}$ with only finitely many nonzero terms. Using the assumption that $\left(\alpha_{m, n}\right)_{m, n=0}^{\infty}$ is diagonal we obtain that

$$
\sum_{m, j, k=0}^{\infty} \alpha_{m+j, n+k} c_{n, j} \bar{c}_{m, k}=\sum_{m=0}^{\infty} \sum_{p, q=0}^{\infty} b_{m+p+q} c_{m, p} c_{n, q} .
$$

Hence (3.8) implies (2.1). Using (2.1) with matrices $\left(c_{j, k}\right)_{j, k=0}^{\infty}$ such that $c_{j, k}=0$ for $j \neq 1$ we see that (2.1) implies (3.5). Finally since (3.6) is equivalent to (2.2), the proof of the lemma is complete.

We recall that a two sided infinite sequence $\left(b_{n}\right)_{n=\infty}^{\infty}$ is called a trigonometric moment sequence if there exists a positive Borel measure $\mu$ on the unit circle

$$
\begin{array}{r}
\boldsymbol{T}=\{z:|z|=1\} \text { such that } b_{n}=\int_{T} e^{-\mathrm{int}} d \mu(t), \\
n=0, \pm 1, \pm 2, \cdots
\end{array}
$$

A sequence $\left(b_{n}\right)_{0}^{\infty}$ is called a power moment sequence of the interval $[a, b]$ on the real line, if there exists a positive Borel measure $\mu$ on $[a, b]$ such that:

$$
b_{n}=\int_{a}^{b} t^{n} d \mu(t), \quad n=0,1,2, \cdots
$$

An obvious application of Theorem 2.1, Lemma 3.1 and Lemma 3.2 now yields:

Proposition 3.3. (a) A sequence $\left(b_{n}\right)_{n=\infty}^{\infty}$ is a trigonometric moment sequence if and only if it satisfies condition (3.2).

(b) A sequence $\left(b_{n}\right)_{n=0}^{\infty}$ is a power moment sequence for the interval $[-1,1]$ if and only if it satisfies (3.3) and (3.4).

(c) A sequence $\left(b_{n}\right)_{n=0}^{\infty}$ is a power moment sequence for the interval $[0,1]$ if and only if it satisfies (3.5) and (3.6).

Part (a) of the proposition is the well known theorem of Herglotz 
on the characterization of trigonometric moment sequences.

Part (b) of the proposition is proved by Akhiezer and Krein [2, p. 35, Theorem 19] for finite sequences of the form $\left(b_{k}\right)_{k=0}^{2 m}$. Since an infinite sequence $\left(b_{k}\right)_{k=0}^{\infty}$ is a power moment sequence for a given interval if and anly if for every $m$ the sequence $\left(b_{k}\right)_{k=0}^{2 m}$ is a finite moment sequence on that interval, it follows that the two conditions are equivalent for infinite sequences.

Part (c) of the proposition is a known theorem, but we are unable to determine its origin. See however Akhieser [1, p. 74].

For the power moment sequences for the interval $[0,1]$ we also have the well known characterization of Housdorff (see [1, p. 74]). It seems that there is not direct way to prove that the two conditions are equivalent.

ACKNowledgement. I would like to thank H. S. Shapiro and A. Shields for a useful conversation concerning the topic of this paper.

\section{REFERENCES}

1. N. I. Akhieser, The classical moment problem, Oliver and Boyd, 1965.

2. N. I. Akhieser and M. G. Krein, Some questions in the theory of moments, Amer. Math. Soc., New York, 1962.

3. J. Bram, Subnormal operators, Duke Math. J., 22 (1955), 75-94.

4. P. R. Halmos, Introduction to Hilbert space, Chelsea 1957.

5. H. Radjavi and P. Rosenthal, Invariant Subspaces, Springer, 1973.

Received January 28, 1975 and in revised form June 12, 1975.

UNIVERSITÉ PARIS-SUd

AND

Department of Mathematics Technion, Haifa, Israel 



\title{
PACIFIC JOURNAL OF MATHEMATICS
}

\section{EDITORS}

RichaRd ARENS (Managing Editor)

University of California

Los Angeles, California 90024

\section{R. A. Beaumont}

University of Washington

Seattle, Washington 98105
J. DUGUNDJI

Department of Mathematics University of Southern California Los Angeles, California 90007

D. Gilbarg and J. Milgram

Stanford University

Stanford, California 94305

\section{ASSOCIATE EDITORS}
E. F. BECKENBACH
B. H. NeUmanN
F. WOLF
K. YoshIDA

\section{SUPPORTING INSTITUTIONS}

\author{
UNIVERSITY OF BRITISH COLUMBIA \\ CALIFORNIA INSTITUTE OF TECHNOLOGY \\ UNIVERSITY OF CALIFORNIA \\ MONTANA STATE UNIVERSITY \\ UNIVERSITY OF NEVADA \\ NEW MEXICO STATE UNIVERSITY \\ OREGON STATE UNIVERSITY \\ UNIVERSITY OF OREGON \\ OSAKA UNIVERSITY
}

\author{
UNIVERSITY OF SOUTHERN CALIFORNIA \\ STANFORD UNIVERSITY \\ UNIVERSITY OF TOKYO \\ UNIVERSITY OF UTAH \\ WASHINGTON STATE UNIVERSITY \\ UNIVERSITY OF WASHINGTON \\ AMERICAN MATHEMATICAL SOCIETY
}

The Supporting Institutions listed above contribute to the cost of publication of this Journal, but they are not owners or publishers and have no responsibility for its content or policies.

Mathematical papers intended for publication in the Pacific Journal of Mathematics should be in typed form or offset-reproduced, (not dittoed), double spaced with large margins. Underline Greek letters in red, German in green, and script in blue. The first paragraph or two must be capable of being used separately as a synopsis of the entire paper. Items of the bibliography should not be cited there unless absolutely necessary, in which case they must be identified by author and Journal, rather than by item number. Manuscripts, in triplicate, may be sent to any one of the editors. Please classify according to the scheme of Math. Reviews, Index to Vol. 39. All other communications should be addressed to the managing editor, or Elaine Barth, University of California, Los Angeles, California, 90024.

The Pacific Journal of Mathematics expects the author's institution to pay page charges, and reserves the right to delay publication for nonpayment of charges in case of financial emergency.

100 reprints are provided free for each article, only if page charges have been substantially paid. Additional copies may be obtained at cost in multiples of 50 .

The Pacific Journal of Mathematics is issued monthly as of January 1966. Regular subscription rate: $\$ 72.00$ a year (6 Vols., 12 issues). Special rate: $\$ 36.00$ a year to individual members of supporting institutions.

Subscriptions, orders for back numbers, and changes of address should be sent to Pacific Journal of Mathematics, 103 Highland Boulevard, Berkeley, California, 94708.

PUBLISHED BY PACIFIC JOURNAL OF MATHEMATICS, A NON-PROFIT CORPORATION

Printed at Kokusai Bunken Insatsusha (International Academic Printing Co., Ltd.), 8-8, 3-chome, Takadanobaba, Shinjuku-ku, Tokyo 160, Japan.

Copyright (C) 1975 by Pacific Journal of Mathematics Manufactured and first issued in Japan 


\section{Pacific Journal of Mathematics}

\section{Vol. 59, No. $2 \quad$ June, 1975}

Aharon Atzmon, A moment problem for positive measures on the unit disc ........

Peter W. Bates and Grant Bernard Gustafson, Green's function inequalities for

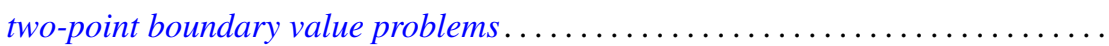

Howard Edwin Bell, Infinite subrings of infinite rings and near-rings ...........

Grahame Bennett, Victor Wayne Goodman and Charles Michael Newman, Norms of

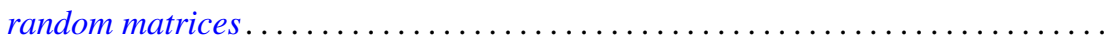

Beverly L. Brechner, Almost periodic homeomorphisms of $E^{2}$ are periodic.........

Beverly L. Brechner and R. Daniel Mauldin, Homeomorphisms of the plane ........

Jia-Arng Chao, Lusin area functions on local fields ......................

Frank Rimi DeMeyer, The Brauer group of polynomial rings ...............

M. V. Deshpande, Collectively compact sets and the ergodic theory of

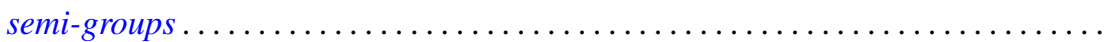

Raymond Frank Dickman and Jack Ray Porter, $\theta$-closed subsets of Hausdorff

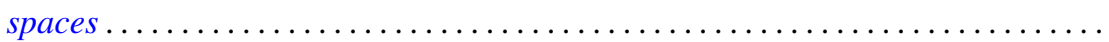

Charles P. Downey, Classification of singular integrals over a local field .......... 407

Daniel Reuven Farkas, Miscellany on Bieberbach group algebras . . . . . . . . . . . .

Peter A. Fowler, Infimum and domination principles in vector lattices . . . . . . . . . .

Barry J. Gardner, Some aspects of T-nilpotence. II: Lifting properties over

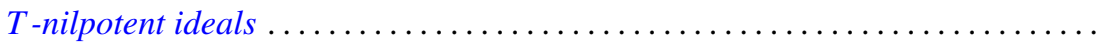

Gary Fred Gruenhage and Phillip Lee Zenor, Metrization of spaces with countable

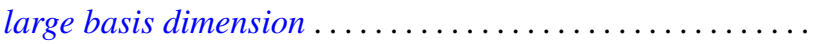

J. L. Hickman, Reducing series of ordinals...

Hugh M. Hilden, Generators for two groups related to the braid group ...

Tom (Roy Thomas Jr.) Jacob, Some matrix transformations on analytic sequence

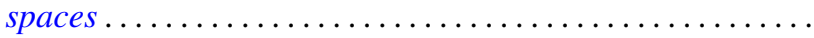

Elyahu Katz, Free products in the category of $k_{w}$-groups . .....

Tsang Hai Kuo, On conjugate Banach spaces with the Radon-Nikodým property...

Norman Eugene Liden, $K$-spaces, their antispaces and related mappings ...

Clinton M. Petty, Radon partitions in real linear spaces ........

Alan Saleski, A conditional entropy for the space of pseudo-Menger maps ....

Michael Singer, Elementary solutions of differential equations .

Eugene Spiegel and Allan Trojan, On semi-simple group algebras. I. . .

Charles Madison Stanton, Bounded analytic functions on a class of open Riemann

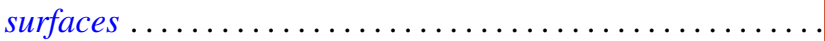

Sherman K. Stein, Transversals of Latin squares and their generalizations ....

Ivan Ernest Stux, Distribution of squarefree integers in non-linear sequences . . .

Lowell G. Sweet, On homogeneous algebras ................

Lowell G. Sweet, On doubly homogeneous algebras .......... .

Florian Vasilescu, The closed range modulus of operators ......

Arthur Anthony Yanushka, A characterization of the symplectic groups $\operatorname{PSp}(2 m, q)$

as rank 3 permutation groups... 